\title{
Vector Meson Production from NLL BFKL
}

\author{
Dmitry Yu. Ivanov ${ }^{1}$ and Alessandro Papa ${ }^{2}$ \\ 1- Sobolev Institute of Mathematics \\ 630090 Novosibirsk - Russia \\ 2- Dipartimento di Fisica, Università della Calabria \\ and INFN - Gruppo Collegato di Cosenza \\ I-87036 Rende - Italy
}

\begin{abstract}
The amplitude for the forward electroproduction of two light vector mesons can be written completely within perturbative QCD in the Regge limit with next-to-leading accuracy, thus providing the first example of a physical application of the BFKL approach at the next-to-leading order. We study in the case of equal photon virtualities the main systematic effects, by considering a different representation of the amplitude and different optimization methods of the perturbative series.
\end{abstract}

\section{Introduction}

In the BFKL approach [2], both in the leading logarithmic approximation (LLA), which means resummation of all terms $\left(\alpha_{s} \ln (s)\right)^{n}$, and in the next-to-leading approximation (NLA), which means resummation of all terms $\alpha_{s}\left(\alpha_{s} \ln (s)\right)^{n}$, the amplitude for a large$s$ hard collision process can be written as the convolution of the Green's function of two interacting Reggeized gluons with the impact factors of the colliding particles.

The Green's function is determined through the BFKL equation. The kernel of the BFKL equation is known now both in the forward [3] and in the non-forward [4] cases. On the other side, impact factors are known with NLA accuracy in a few cases: colliding partons [5], forward jet production [6] and forward transition from a virtual photon $\gamma^{*}$ to a light neutral vector meson $V=\rho^{0}, \omega, \phi[7]$. The most important impact factor for phenomenology, the $\gamma^{*} \rightarrow \gamma^{*}$ impact factor, is calling for a rather long calculation, which seems to be close to completion now $[8,9]$.

The $\gamma^{*} \rightarrow V$ forward impact factor can be used together with the NLA BFKL forward Green's function to build, completely within perturbative QCD and with NLA accuracy, the amplitude of the $\gamma^{*} \gamma^{*} \rightarrow V V$ reaction. This amplitude provides us with an ideal theoretical laboratory for the investigation of several open questions in the BFKL approach. Besides, this process can be studied experimentally at the future at ILC, see Refs. [10].

\section{Representations of the NLA amplitude}

The process under consideration is the production of two light vector mesons $\left(V=\rho^{0}, \omega, \phi\right)$ in the collision of two virtual photons, $\gamma^{*}(p) \gamma^{*}\left(p^{\prime}\right) \rightarrow V\left(p_{1}\right) V\left(p_{2}\right)$. Here, neglecting the meson mass $m_{V}, p_{1}$ and $p_{2}$ are taken as Sudakov vectors satisfying $p_{1}^{2}=p_{2}^{2}=0$ and $2\left(p_{1} p_{2}\right)=s$; the virtual photon momenta are instead $p=\alpha p_{1}-Q_{1}^{2} /(\alpha s) p_{2}$ and $p^{\prime}=$ $\alpha^{\prime} p_{2}-Q_{2}^{2} /\left(\alpha^{\prime} s\right) p_{1}$, so that the photon virtualities turn to be $p^{2}=-Q_{1}^{2}$ and $\left(p^{\prime}\right)^{2}=-Q_{2}^{2}$. We consider the kinematics when $s \gg Q_{1,2}^{2} \gg \Lambda_{Q C D}^{2}$ and $\alpha=1+Q_{2}^{2} / s+\mathcal{O}\left(s^{-2}\right), \alpha^{\prime}=1+$ $Q_{1}^{2} / s+\mathcal{O}\left(s^{-2}\right)$. In this case vector mesons are produced by longitudinally polarized photons in the longitudinally polarized state [7]. Other helicity amplitudes are power suppressed, with a suppression factor $\sim m_{V} / Q_{1,2}$. We will discuss here the amplitude of the forward 
scattering, i.e. when the transverse momenta of produced $V$ mesons are zero or when the variable $t=\left(p_{1}-p\right)^{2}$ takes its maximal value $t_{0}=-Q_{1}^{2} Q_{2}^{2} / s+\mathcal{O}\left(s^{-2}\right)$.

The NLA forward amplitude can be written as a spectral decomposition on the basis of eigenfunctions of the LLA BFKL kernel:

$$
\begin{aligned}
& \frac{\operatorname{Im}_{s}\left(\mathcal{A}_{\mathrm{e} x p}\right)}{D_{1} D_{2}}=\frac{s}{(2 \pi)^{2}} \int_{-\infty}^{+\infty} d \nu\left(\frac{s}{s_{0}}\right)^{\bar{\alpha}_{s}\left(\mu_{R}\right) \chi(\nu)+\bar{\alpha}_{s}^{2}\left(\mu_{R}\right)\left(\bar{\chi}(\nu)+\frac{\beta_{0}}{8 N_{c}} \chi(\nu)\left[-\chi(\nu)+\frac{10}{3}\right]\right)} \alpha_{s}^{2}\left(\mu_{R}\right) c_{1}(\nu) c_{2}(\nu) \\
& \times\left[1+\bar{\alpha}_{s}\left(\mu_{R}\right)\left(\frac{c_{1}^{(1)}(\nu)}{c_{1}(\nu)}+\frac{c_{2}^{(1)}(\nu)}{c_{2}(\nu)}\right)+\bar{\alpha}_{s}^{2}\left(\mu_{R}\right) \ln \left(\frac{s}{s_{0}}\right) \frac{\beta_{0}}{8 N_{c}} \chi(\nu)\left(i \frac{d \ln \left(\frac{c_{1}(\nu)}{c_{2}(\nu)}\right)}{d \nu}+2 \ln \left(\mu_{R}^{2}\right)\right)\right] .
\end{aligned}
$$

Here the bulk of NLA kernel corrections are exponentiated, $\bar{\alpha}_{s}=\alpha_{s} N_{c} / \pi$ and $D_{1,2}=$ $-4 \pi e_{q} f_{V} /\left(N_{c} Q_{1,2}\right)$, where $f_{V}$ is the meson dimensional coupling constant $\left(f_{\rho} \approx 200 \mathrm{MeV}\right)$ and $e_{q}$ should be replaced by $e / \sqrt{2}, e /(3 \sqrt{2})$ and $-e / 3$ for the case of $\rho^{0}, \omega$ and $\phi$ meson production, respectively. Two scales enter the expression (1), the renormalization scale $\mu_{R}$ and the scale for energy $s_{0}$.

Alternatively, the amplitude can be expressed as a series:

$$
\begin{aligned}
\frac{Q_{1} Q_{2}}{D_{1} D_{2}} \frac{\operatorname{Im}_{s}\left(\mathcal{A}_{\text {series }}\right)}{s} & =\frac{1}{(2 \pi)^{2}} \alpha_{s}\left(\mu_{R}\right)^{2} \\
& \times\left[b_{0}+\sum_{n=1}^{\infty} \bar{\alpha}_{s}\left(\mu_{R}\right)^{n} b_{n}\left(\ln \left(\frac{s}{s_{0}}\right)^{n}+d_{n}\left(s_{0}, \mu_{R}\right) \ln \left(\frac{s}{s_{0}}\right)^{n-1}\right)\right] .
\end{aligned}
$$

The $b_{n}$ coefficients are determined by the kernel and the impact factors in LLA, while the $d_{n}$ coefficients depend also on the NLA corrections to the kernel and to the impact factors. We refer to Ref. [11] for the details of the derivation and for the definition of the functions entering these expressions.

\section{$3 \quad$ Numerical results}

In Ref. [11] we presented some numerical results for the amplitude given in Eq. (2) for the $Q_{1}=Q_{2} \equiv Q$ kinematics, i.e. in the "pure" BFKL regime. We found that the $d_{n}$ coefficients are negative and increasingly large in absolute values as the perturbative order increases, making evident the need of an optimization of the perturbative series. We adopted the principle of minimal sensitivity (PMS) [12], by requiring the minimal sensitivity of the predictions to the change of both the renormalization and the energy scales, $\mu_{R}$ and $s_{0}$. We considered the amplitude for $Q^{2}=24 \mathrm{GeV}^{2}$ and $n_{f}=5$ and studied its sensitivity to variation of the parameters $\mu_{R}$ and $Y_{0}=\ln \left(s_{0} / Q^{2}\right)$. We could see that for each value of $Y=\ln \left(s / Q^{2}\right)$ there are quite large regions in $\mu_{R}$ and $Y_{0}$ where the amplitude is practically independent on $\mu_{R}$ and $Y_{0}$ and we got for the amplitude a smooth behaviour in $Y$ (see the curve labeled "series - PMS" in Figs. 1 and 2). The optimal values turned out to be $\mu_{R} \simeq 10 Q$ and $Y_{0} \simeq 2$, quite far from the kinematical values $\mu_{R}=Q$ and $Y_{0}=0$. These "unnatural" values probably mimic large unknown NNLA corrections. 

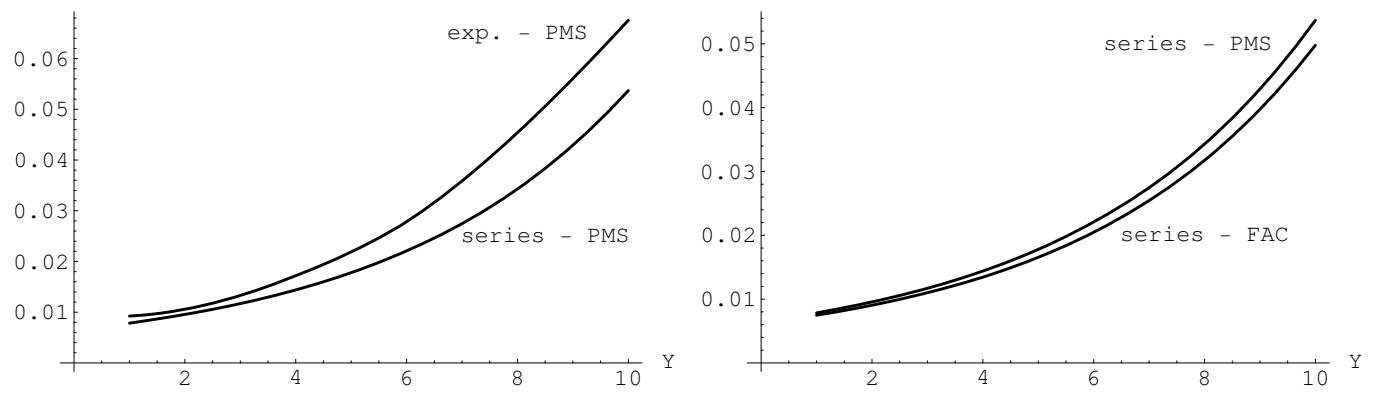

Figure 1: $\operatorname{Im}_{s}(\mathcal{A}) Q^{2} /\left(s D_{1} D_{2}\right)$ as a function of $Y$ at $Q^{2}=24 \mathrm{GeV}^{2}\left(n_{f}=5\right)$ : (left) series representation with PMS and "exponentiated" representation with PMS, (right) series representation with PMS and with FAC.

As an estimation of the systematic effects in our determination, we considered also the "exponentiated" representation of the amplitude, Eq. (1), and different optimization methods. For more details on the following, see Ref. [13].

At first, we compare the series and the "exponentiated" determinations using in both case the PMS method. The optimal values of $\mu_{R}$ and $Y_{0}$ for the "exponentiated" amplitude are quite similar to those obtained in the case of the series representation, with only a slight decrease of the optimal $\mu_{R}$. Fig. 1 (left) shows that the two determinations are in good agreement at the lower energies, but deviate increasingly for large values of $Y$. It should be stressed, however, that the applicability domain of the BFKL approach is determined by the condition $\bar{\alpha}_{s}\left(\mu_{R}\right) Y \sim 1$ and, for $Q^{2}=24 \mathrm{GeV}^{2}$ and for the typical optimal values of $\mu_{R}$, one gets from this condition $Y \sim 5$. Around this value the discrepancy between the two determinations is within a few percent.

As a second check, we changed the optimization method and applied it both to the series and to the "exponentiated" representation. The method considered is the fast apparent convergence (FAC) method [14], whose strategy, when applied to a usual perturbative expansion, is to fix the renormalization scale to the value for which the highest order correction term is exactly zero. In our case, the application of the FAC method requires an adaptation, for two reasons: the first is that we have two energy parameters in the game, $\mu_{R}$ and $Y_{0}$, the second is that, if only strict NLA corrections are taken, the amplitude does not depend at all on these parameters. For details about the application of this method, we refer to [13]. Here, we merely show the results: the FAC method applied to the series representation (see Fig. 1 (right)) and to the exponentiated representation (see Fig. 2 (left)) gives results in nice agreement with those from the PMS method applied to the series representation, over the whole energy range considered.

Another popular optimization method is the Brodsky-Lepage-Mackenzie (BLM) one [15], which amounts to perform a finite renormalization to a physical scheme and then to choose the renormalization scale in order to remove the $\beta_{0}$-dependent part. We applied this method only to the series representation, Eq. (2). The result is compared with the PMS method in Fig. 2 (right) (for details, see Ref. [13]). 

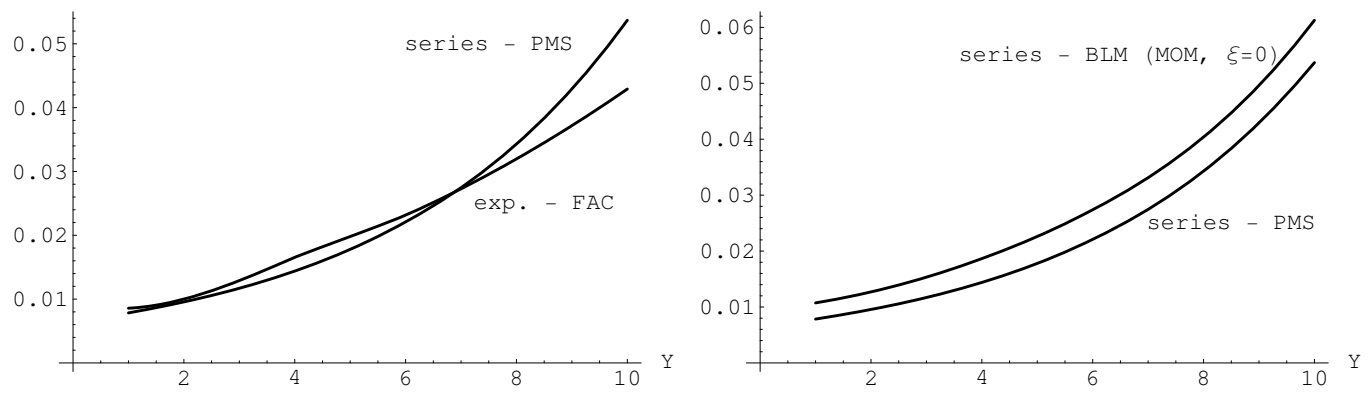

Figure 2: $\operatorname{Im}_{s}(\mathcal{A}) Q^{2} /\left(s D_{1} D_{2}\right)$ as a function of $Y$ at $Q^{2}=24 \mathrm{GeV}^{2}\left(n_{f}=5\right)$ : (left) series representation with PMS and "exponentiated" representation with FAC, (right) series representation with PMS and with BLM.

\section{Acknowledgments}

The work of D.I. was partially supported by grants RFBR-05-02-16211, NSh-5362.2006.2.

\section{References}

[1] Slides: http://indico. cern. ch/contributionDisplay. py? contribId=76\&sessionId $=7 \&$ conf Id $=9499$

[2] V.S. Fadin, E.A. Kuraev, L.N. Lipatov, Phys. Lett. B60 50 (1975); E.A. Kuraev, L.N. Lipatov and V.S. Fadin, Zh. Eksp. Teor. Fiz. 71840 (1976) [Sov. Phys. JETP 44443 (1976)]; 72377 (1977) [45 199 (1977)]; Ya.Ya. Balitskii and L.N. Lipatov, Sov. J. Nucl. Phys. 28822 (1978).

[3] V.S. Fadin and L.N. Lipatov, Phys. Lett. B429 127 (1998); M. Ciafaloni and G. Camici, Phys. Lett. B430 349 (1998).

[4] V.S. Fadin and R. Fiore, Phys. Lett. B610 61 (2005) [Erratum-ibid. B621 61 (2005)]; Phys. Rev. D72 014018 (2005).

[5] V.S. Fadin, R. Fiore, M.I. Kotsky and A. Papa, Phys. Rev. D61 094005 (2000); Phys. Rev. D61 094006 (2000); M. Ciafaloni and G. Rodrigo, JHEP 0005042 (2000).

[6] J. Bartels, D. Colferai and G.P. Vacca, Eur. Phys. J. C24 83 (2002); Eur. Phys. J. C29 235 (2003).

[7] D.Yu. Ivanov, M.I. Kotsky and A. Papa, Eur. Phys. J. C38 195 (2004); Nucl. Phys. (Proc. Suppl.) 146 117 (2005).

[8] J. Bartels, S. Gieseke and C.F. Qiao, Phys. Rev. D63 056014 (2001)[Erratum-ibid. D65 079902 (2002)]; J. Bartels, S. Gieseke and A. Kyrieleis, Phys. Rev. D65 014006 (2002); J. Bartels, D. Colferai, S. Gieseke and A. Kyrieleis, Phys. Rev. D66 094017 (2002); J. Bartels, Nucl. Phys. (Proc. Suppl.) 116 (2003); J. Bartels and A. Kyrieleis, Phys. Rev. D70 114003 (2004); V.S. Fadin, D.Yu. Ivanov and M.I. Kotsky, Phys. Atom. Nucl. 651513 (2002) [Yad. Fiz. 651551 (2002)]; V.S. Fadin, D.Yu. Ivanov and M.I. Kotsky, Nucl. Phys. B658 156 (2003).

[9] G. Chachamis and J. Bartels, "NLO Photon Impact Factor: Present Status and Outlook", PoS(DIFF2006)026.

[10] R. Enberg, B. Pire, L. Szymanowski and S. Wallon, Eur. Phys. J. C45 759 (2006); M. Segond, L. Szymanowski and S. Wallon, arXiv:hep-ph/0703166 (2007).

[11] D.Yu. Ivanov and A. Papa, Nucl. Phys. B 732183 (2006).

[12] P.M. Stevenson, Phys. Lett. B100 61 (1981); Phys. Rev. D23 2916 (1981).

[13] D.Yu. Ivanov and A. Papa, Eur. Phys. J. C 49947 (2007).

[14] G. Grunberg, Phys. Lett. B95 70 (1980) [Erratum-ibid. B110 501 (1982)]; ibid. B114 271 (1982); Phys. Rev. D29 2315 (1984).

[15] S.J. Brodsky, G.P. Lepage, P.B. Mackenzie, Phys. Rev. D28 228 (1983). 